\title{
Bonded Excimer in Stacked Cytosines: A Semiclassical Simulation Study
}

\author{
Weifeng Wu, ${ }^{1}$ Shuai Yuan, ${ }^{1}$ Jiajie She, ${ }^{1}$ Yusheng Dou, ${ }^{2}$ and Roland E. Allen ${ }^{3}$ \\ ${ }^{1}$ Institute of Computational Chemistry, Chongqing University of Posts and Telecommunications, Chongqing, 400065, China \\ ${ }^{2}$ Department of Physical Sciences, Nicholls State University, P.O. Box 2022, Thibodaux, LA 70310, USA \\ ${ }^{3}$ Department of Physics and Astronomy, Texas A\&M University, College Station, TX 77843-4242, USA
}

Correspondence should be addressed to Shuai Yuan; yuanshuai@cqupt.edu.cn and Yusheng Dou; yusheng.dou@nicholls.edu

Received 13 May 2014; Accepted 21 July 2014

Academic Editor: Fuli Li

Copyright (C) 2015 Weifeng Wu et al. This is an open access article distributed under the Creative Commons Attribution License, which permits unrestricted use, distribution, and reproduction in any medium, provided the original work is properly cited.

\begin{abstract}
The formation of a covalent bond between two stacked cytosines, one of which is excited by an ultrafast laser pulse, was studied by semiclassical dynamics simulations. The results show that a bonded excimer is created, which sharply lowers the energy gap between the LUMO and HOMO and consequently facilitates the deactivation of the electronically excited molecule. This is different from the case of two stacked adenines, where the formation of a covalent bond alters the nonadiabatic deactivation mechanism in two opposite ways. It lowers the energy gap and consequently leads to the coupling between the HOMO and LUMO levels, thus enhancing the deactivation of the electronically excited molecule. On the other hand, it leads to restriction of the deformation vibration of the pyrimidine in the excited molecule, because of a steric effect, and this delays the deactivation process of the excited adenine molecule with return to the electronic ground state.
\end{abstract}

\section{Introduction}

The mechanism and, therefore, the time scale, for electronically excited DNA bases to decay to their ground state, are greatly affected by base stacking. Decay of a monomer DNA base occurs on a subpicosecond time scale through a conical intersection between the lowest electronic excited state and the ground state [1-4]. Excited stacked bases show different nonradiative decay pathways [5]. Poorly stacked bases show a monomer-like decay pathway, while strongly stacked bases exhibit a slower decay channel. The slow decay channel is explained by the formation of an excimer between an excited base and a ground state neighboring base. Excimers have a longer lifetime with substantial charge transfer character [5-11].

Two stacked molecules may form a chemical bond, resulting in a bonded excimer. It has been reported [12, 13] that the formation of a bonded excimer plays a role in the rapid charge-transfer quenching reaction of a singlet excited state formed between cyanoaromatic electron acceptors and pyridine. The density functional theory (DFT) calculations suggest $[12,13]$ that the formation of a covalent bond between two stacked molecules lowers the energy of charge-transfer state significantly. One question that arises is whether the formation of a chemical bond between two adjacent bases also plays a rule in the nonadiabatic decay process of stacked DNA bases. Since the formation of a chemical bond is considerably exothermic, an excimer (or exciplex if two bases are not identical) already formed in stacked DNA bases may go further to form a bonded excimer (or exciplex).

Our previous semiclassical dynamics simulation [14] for the effect of $\pi$-stacking on the decay channels of an adenine molecule excited by an ultrafast laser pulse finds the formation a bonded excimer between two stacked bases after laser excitation. The formation of the bonded excimer shrinks the energy gap between the HOMO and LUMO energy levels and, thus, favors the nonadiabatic decay process. On the other hand, the formation of the bonded excimer also results in steric hindrance of the excited adenine, which restricts the molecular deformation that leads to nonradiative decay to the ground state. The simulation results, thus, lead to the conclusion that the formation of a covalent bond between 
two stacked bases alters the electronic structure but also induces the steric effect, with both mechanisms affecting the nonradiative decay of excited DNA base molecules.

The work reported here provides evidence that the formation of a covalent bond dose in fact plays a role in nonadiabatic decay of electronic excitation in two stacked cytosine molecules. Our simulations use a semiclassical dynamics technique, in which all the degrees of freedom of the system are included in the calculations and the forces acting on each nucleus are calculated on-the-fly. The results show that a covalent bond is formed between two adjacent bases in some trajectories after laser excitation and provide fundamental information for understanding this important phenomenon in the nonradiative decay of stacked-bases.

\section{Methodology}

Our technique here is semiclassical electron-radiation-ion dynamics (SERID). A detailed description of this method has been published elsewhere $[15,16]$ and only a brief summary is presented here. The valence electrons are treated with a quantum mechanical approach while both the radiation field and the motion of the nuclei are treated classically. The one-electron states are updated by solving the timedependent Schrödinger equation at each time step (usually 0.05 femtosecond in duration) in a nonorthogonal basis:

$$
i \hbar \frac{\partial \Psi_{j}}{\partial t}=\mathbf{S}^{-1} \cdot \mathbf{H} \cdot \Psi_{j}
$$

where $\mathbf{S}$ represents the overlap matrix of the atomic orbitals. The vector potential $\mathbf{A}$ of the radiation field is included in the electronic Hamiltonian via the time-dependent Peierls substitution [17]:

$$
\mathbf{H}_{a b}\left(X-X^{\prime}\right)=\mathbf{H}_{a b}^{0}\left(X-X^{\prime}\right) \exp \left\{\frac{i q}{\hbar c} \mathbf{A} \cdot\left(X-X^{\prime}\right)\right\},
$$

where $\mathbf{H}_{a b}\left(X-X^{\prime}\right)$ is the Hamiltonian matrix element for basis functions $a$ and $b$ on atoms atpositions $X$ and $X^{\prime}$, respectively, and $q=-e$ is the charge of the electron.

The forces acting on nuclei or ions are computed by the Ehrenfest equation in an "on-the-fly" approach:

$$
M_{l} \frac{d^{2} X_{l \alpha}}{d t^{2}}=-\frac{1}{2} \sum_{j} \Psi_{j}^{+} \cdot\left\{\frac{\partial \mathbf{H}}{\partial X_{l \alpha}}-i \hbar \frac{\partial \mathbf{S}}{\partial X_{l \alpha}} \cdot \frac{\partial}{\partial t}\right\} \cdot \Psi_{j}-\frac{\partial U_{\text {rep }}}{\partial X_{l \alpha}}
$$

where $U_{\text {rep }}$ is the effective nuclear-nuclear repulsive potential and $X_{l \alpha}=\left\langle\widehat{X}_{l \alpha}\right\rangle$ is the expectation value of the timedependent Heisenberg operator for the $\alpha$ coordinate of the nucleus labeled by $l$ (with $\alpha=x, y$, or $z$ ).

The Hamiltonian matrix $H$, overlap matrix $S$, and effective nuclear-nuclear repulsive potential $U_{\text {rep }}$ are calculated in the density-functional tight-binding approximation [18, 19]. These quantities are functions only of the nuclear distance and the results from the calculations for a dimer can be tabulated and employed in the time-dependent calculations.
The basis functions used in the present simulations are the 1 s atomic orbital of $H$ and the valence $s$ and $p$ orbitals of $C$ and $N$ (spin up and spin down states are not distinguished). In this approach, the electronic energy of a molecule can be written as

$$
E_{\mathrm{elec}}=\sum_{i=\mathrm{occ}} n_{i} \varepsilon_{i}+\sum_{\alpha>\beta} U_{\mathrm{rep}}\left(\left|X_{\alpha}-X_{\beta}\right|\right),
$$

where $\varepsilon_{i}$ and $\mathbf{n}_{\mathbf{i}}$ are the eigenvalue and occupation number of Kohn-Sham orbital i. The first summation goes over all occupied orbitals. The effective repulsive potential $U_{\text {rep }}\left(\mid X_{\alpha}-\right.$ $\left.X_{\beta} \mid\right)$ is a function of the interatom distance.

We have previously applied the SERID technique to study nonadiabatic decay in adenine $[20,21]$, photodissociation of the cyclobutane thymine dimer $[22,23]$, photoinduced dimerization of thymine [24], and the cytosine dimer [25], and the results were found to be consistent with experimental observations. A limitation of this method is that the simulation trajectory moves along a path produced by averaging over all the terms in the Born-Oppenheimer expansion $[26-30]$

$$
\Psi^{\text {total }}\left(X_{n}, x_{e}, t\right)=\sum_{i} \Psi_{i}^{n}\left(X_{n}, t\right) \Psi_{i}^{e}\left(x_{e}, X_{n}\right)
$$

rather than following the time evolution of a single potential energy surface [28-30] (here, $X_{n}$ and $x_{e}$ represent the sets of nuclear and electronic coordinates, respectively, and the $\Psi_{i}^{e}$ are eigenstates of the electronic Hamiltonian at fixed $X_{n}$ ). The strengths of the present approach are that it retains all of the $X_{n}$ nuclear degrees of freedom and it incorporates both the excitation due to a laser pulse and the subsequent deexcitation at an avoided crossing near a conical intersection.

A dynamical simulation was run for ground-state cytosine at room temperature $(298 \mathrm{~K})$ for $2000 \mathrm{fs}$. A second cytosine molecule of the same geometry was oriented such that $\mathrm{C}_{5}-\mathrm{C}_{5}^{\prime}=3.45 \AA, \mathrm{C}_{6}-\mathrm{C}_{6}^{\prime}=3.43 \AA$, and $\mathrm{C}_{5}-\mathrm{C}_{6}-\mathrm{C}_{6}^{\prime}-\mathrm{C}_{5}^{\prime}$ $=26.4^{\circ}$; primed labels refer to atoms of the second groundstate cytosine molecule. The simulation was continued for another $2000 \mathrm{fs}$. Twenty structures at $200 \mathrm{fs}$ intervals were recorded. The structure at $1000 \mathrm{fs}$ is shown in Figure 1, where the two molecules are stacked such that the interatomic distances $\mathrm{C}_{5}-\mathrm{C}_{5}^{\prime}$ and $\mathrm{C}_{6}-\mathrm{C}_{6}^{\prime}$ are 3.85 and $3.90 \AA$, respectively, and the dihedral angle $C_{4}-C_{5}-C_{5}^{\prime}-C_{4}^{\prime}$ is $36.1^{\circ}$. Each of the twenty structures was used as the starting geometry for a simulated trajectory initiated by laser excitation. In the following discussion, the excited cytosine molecule will be referred to as molecule $\mathrm{C}$ and the other unexcited molecule as $\mathrm{C}^{\prime}$.

A $25 \mathrm{fs}$ FWHM laser pulse with a Gaussian profile and photon energy of $4.1 \mathrm{eV}$ was used in the simulations. The selected photon energy corresponds to the energy gap between the LUMO and HOMO, as calculated with the present approximations. A fluence in the range 100 to $300 \mathrm{~J} / \mathrm{m}^{2}$ was used, so that the forces produced do not break any bonds. The simulation was run at the selected laser pulse for 500 trajectories and only a typical trajectory is reported in this paper because other trajectories have shown similar properties. 


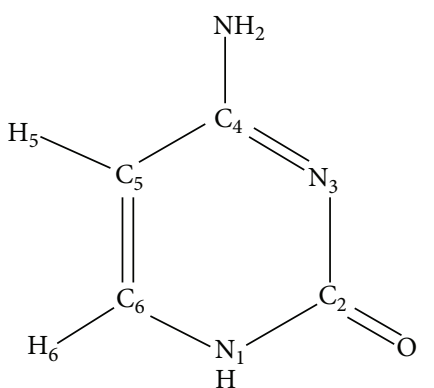

(a)

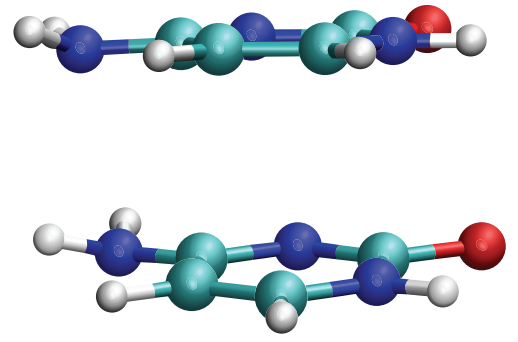

(b)

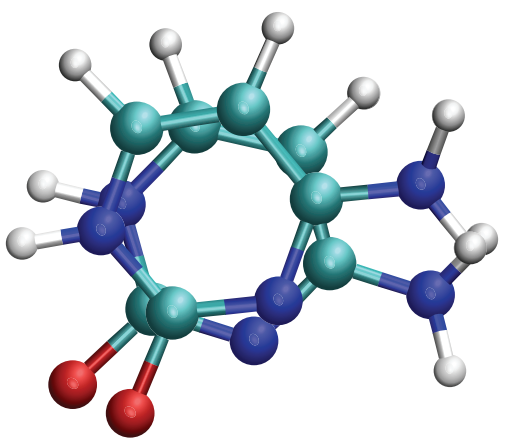

(c)

FIGURE 1: Structure and atomic labeling for the stacked cytosine molecules.
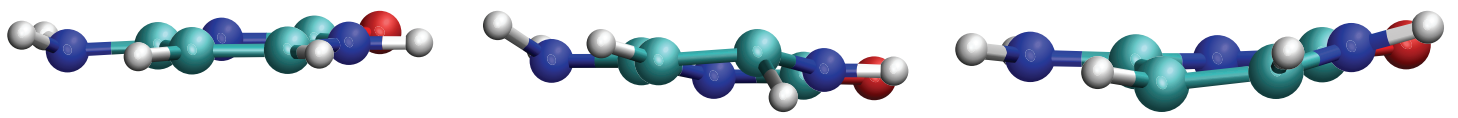

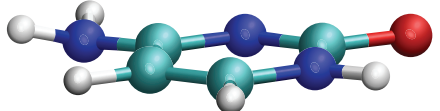

(a)

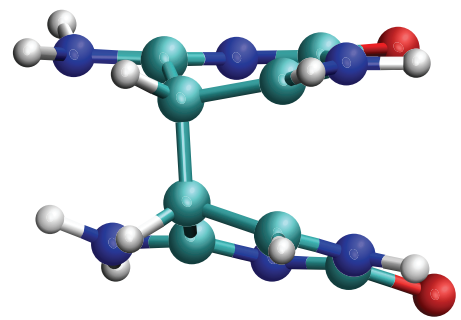

(d)

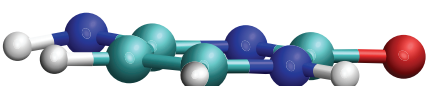

(b)

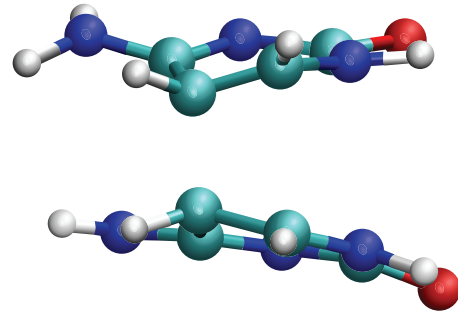

(e)

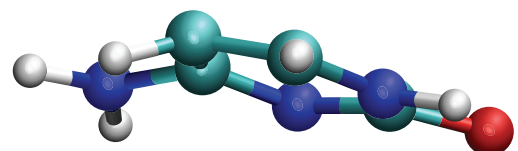

(c)

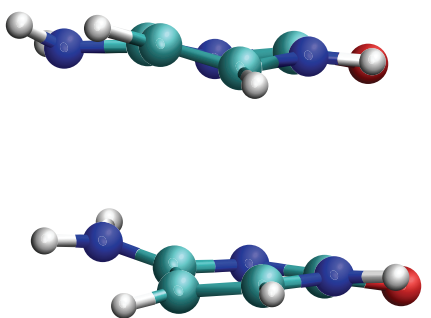

(f)

Figure 2: Snapshots taken from the simulation of two stacked cytosine molecules at (a) 0, (b) 244, (c) 923, (d) 964, (e) 993, and (f) 1082 fs.

\section{Results and Discussion}

The results for a representative trajectory are shown in Figures 2 through 5. Six snapshots from the simulation are shown in Figure 2. Starting from the equilibrated geometry, the bottom molecule is electronically excited by the laser pulse (fluence $=44.01 \mathrm{~J} / \mathrm{m}^{2}$ ). The excited molecule undergoes an out-of-plane vibration of the $\mathrm{N}$ atom from $244 \mathrm{fs}$ to $923 \mathrm{fs}$ (Figures 2(b) and 2(c)). The unexcited molecule also becomes active in vibration because of the stacking interaction. The planar structure of both molecules shows a significant deformation at the $C_{5}$ and $C_{5}^{\prime}$ sites after $923 \mathrm{fs}$ (Figure 2(c)) and a chemical bond has been formed between these atoms after $923 \mathrm{fs}$ (Figure 2(d)). After $993 \mathrm{fs}$, the bond has been broken and two stacked molecules move away from each other (Figure 2(f)).

The variations of the distance between the $\mathrm{C}_{5}$ and $\mathrm{C}_{5}^{\prime}$ atoms with time are shown in Figure 3 which confirms that the formation of a chemical bond with a length of $\sim 1.5 \AA$ takes place at about $964 \mathrm{fs}$; the bond stays stable for a short time and is then dissociated. Figure 3 also shows the changes in the distance between the $C_{5}$ and $C_{5}^{\prime}$ atoms, providing evidence for the potential formation of the cyclobutane dimer.

The variations with time of the HOMO and LUMO levels of two stacked molecules are presented in Figure 4(a), and the time-dependent population of these frontier molecular orbitals is plotted in Figure 4(b). The gap between the HOMO and LUMO initially is about $4.5 \mathrm{eV}$ and becomes less than $2.5 \mathrm{eV}$ after $100 \mathrm{fs}$, when the laser pulse ends. There are two avoided crossings at 923 and $993 \mathrm{fs}$ when the gap is about 0.06 and $0.18 \mathrm{eV}$, respectively. The energies of the HOMO and LUMO move back to approximately their initial values after that. It is seen from Figure 4(b) that the laser excitation promotes about 1 electron from the HOMO to the LUMO, and the couplings between the HOMO and LUMO at $920 \mathrm{fs}$ and $\sim 990 \mathrm{fs}$ lead to the transfer of electrons from LUMO to 


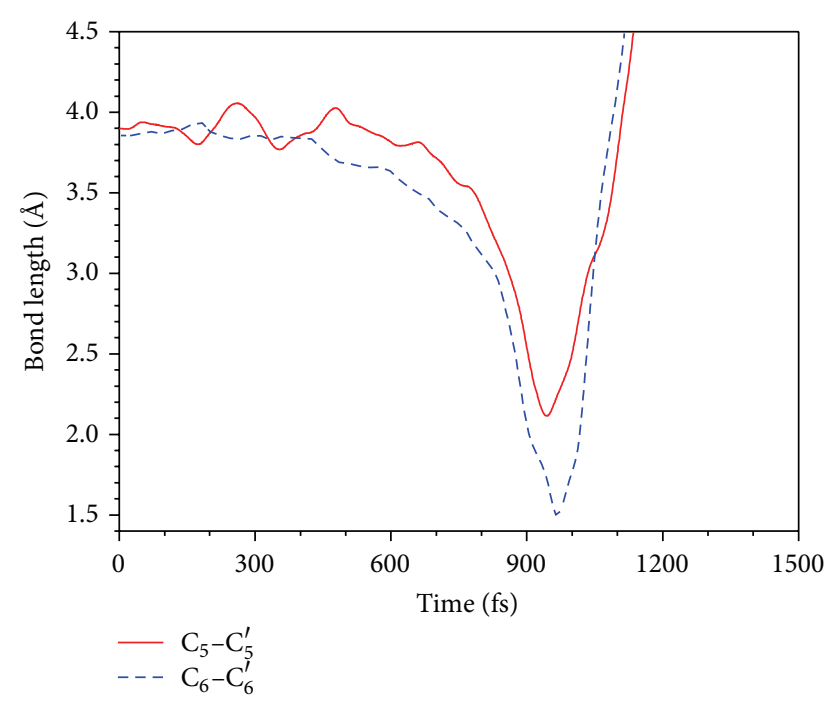

FIgURE 3: The variations with time of the lengths between the $\mathrm{C}_{5}$ and $\mathrm{C}_{5}^{\prime}$ atoms and the $\mathrm{C}_{6}$ and $\mathrm{C}_{6}^{\prime}$ atoms in two stacked cytosine molecules.

HOMO. The initial promotion of electrons from the HOMO to the LUMO brings molecule $\mathrm{C}$ to an electronically excited state and excites some vibrational modes, for example, the out-of-plane vibration of $-\mathrm{NH}_{2}$, as seen in Figure 2. The decrease in the energy gap between the HOMO and LUMO after laser excitation is attributed to the changes in the structure of stacked bases molecules due to the excitation of different vibrational modes. Further diminution of the energy gap from $\sim 850 \mathrm{fs}$ to $\sim 1050 \mathrm{fs}$ is a result of the strong interaction between the two stacked molecules. Specifically, the avoided crossings at $923 \mathrm{fs}$ and $993 \mathrm{fs}$ are associated with the interaction between the $\mathrm{C}_{6}$ and $\mathrm{C}_{6}^{\prime}$ atoms and the $\mathrm{C}_{5}$ and $\mathrm{C}_{5}^{\prime}$ atoms, which leads to the formation of the chemical bond between two adjacent molecules. The avoided crossings result in the transfer of electrons from the LUMO to HOMO, which eventually brings excited molecules to the electronic ground state.

Figure 5 shows the variation with time of the $\mathrm{C}_{5}-\mathrm{C}_{6}$ and $\mathrm{C}_{5}^{\prime}-\mathrm{C}_{6}^{\prime}$ bond lengths. The $\mathrm{C}_{5}-\mathrm{C}_{6}$ bond stretches from an initial value of $\sim 1.33 \AA$ to $\sim 1.47 \AA$ after laser excitation. It remains at this length until $\sim 1000 \mathrm{fs}$ and then returns to approximately its initial length. On the other hand, the same bond for the unexcited molecule only vibrates about its initial length for the entire simulation time. The $\mathrm{C}_{4}^{\prime}-\mathrm{C}_{5}^{\prime}$ bond length sharply rises up from $\sim 1.37 \AA$ to $\sim 1.48 \AA$ after 900 fs and suddenly drops down to its initial value after $1000 \mathrm{fs}$.

The changes in the $\mathrm{C}_{5}-\mathrm{C}_{6}$ bond length is due to excitation and deexcitation of the cytosine molecule, while the changes in the $\mathrm{C}_{5}^{\prime}-\mathrm{C}_{6}^{\prime}$ bond length between $\sim 900 \mathrm{fs}$ and $\sim 1000 \mathrm{fs}$ can be attributed to the formation of the bonded excimer between two adjacent cytosine molecules.

The simulation results presented in this section suggest the photophysical processes described below. By raising about 1 electron from the HOMO to the higher level frontier molecular orbitals, the laser pulse promotes the top molecule from the electronic ground state to a low-lying energy state $\pi \pi^{*}$. In this electronically excited state, the $\mathrm{C}_{5}-\mathrm{C}_{6}$ bond becomes longer. The interaction between excited base and unexcited base due to the stacking effect attracts the two molecules closer to each other. At about $900 \mathrm{fs}$, a covalent bond is formed between the $\mathrm{C}_{5}$ and $\mathrm{C}_{5}^{\prime}$ atoms, leading to the formation of the bonded excimer state. The formation of the covalent bond between two cytosine molecules breaks the $\pi-\pi$ conjugation of the unexcited cytosine. This lengthens the $\mathrm{C}_{5}^{\prime}-\mathrm{C}_{6}^{\prime}$ bond and shortens the $\mathrm{C}_{4}^{\prime}-\mathrm{C}_{5}^{\prime}$ and $\mathrm{C}_{6}^{\prime}-\mathrm{N}_{1}^{\prime}$ bonds (which are not shown here). The energy gap between the LUMO and HOMO decreases sharply from $900 \mathrm{fs}$ to $1100 \mathrm{fs}$, suggesting that the bonded excimer state is substantially lower than the unbonded state in energy. After $900 \mathrm{fs}$, the system thus evolves as a bonded excimer state. It then enters the vicinity of the conical intersection twice, at $923 \mathrm{fs}$ and $993 \mathrm{fs}$, respectively, and it decays to the ground state through a channel resulting from the coupling between the LUMO and HOMO. The coupling occurs primarily because of the formation of the covalent bond between two adjacent molecules. The return to the electronic ground state of the excited molecule is confirmed by the changes in the bond lengthens described above.

The formation of a covalent bond between two adjacent molecules has also been suggested in stacked adenines [14]. It was found by semiclassical dynamics simulations that the bond formation and breaking greatly alter the coupling, leading to decays of the excited system to the ground state and suggesting the involvement of the bonded excimer in the nonradiative decay process for stacked bases. The formation of a covalent bond between two stacked adenine molecules has two opposite effects on nonradiative decay. First it stabilizes the excimer state, which is evidenced by the decrease in the LUMO energy level and thus makes a positive contribution to the excited state lifetime. On the other hand, it limits the conformational flexibility of each molecule. For example, this bond hinders the out-of-plane deformations of the pyrimidine ring $[21,22]$ and, therefore, limits the freedom to achieve the deformation required for accessing the deactivating conical intersections.

Compared to adenine, the cytosine conformation exhibits only a very limited steric effect. This may, at least partially, explain the fact that stacked cytosines have a shorter deactivation time scale than stacked adenine.

\section{Conclusion}

The present investigation focuses on the formation of covalent bond between two stacked cytosines after laser excitation, which strongly affects the HOMO and LUMO energy levels and dominates their couplings. It is these couplings that lead to nonadiabatic decay of the excited system to the electronic ground state. The results for stacked cytosines are different from those for stacked adenines, where formation of a covalent bond affects nonadiabatic decay of the excited system in two different ways. It stabilizes the bonded excimer state between two stacked adenine molecules and also leads to a steric effect which inhibits nonradiative decay. 


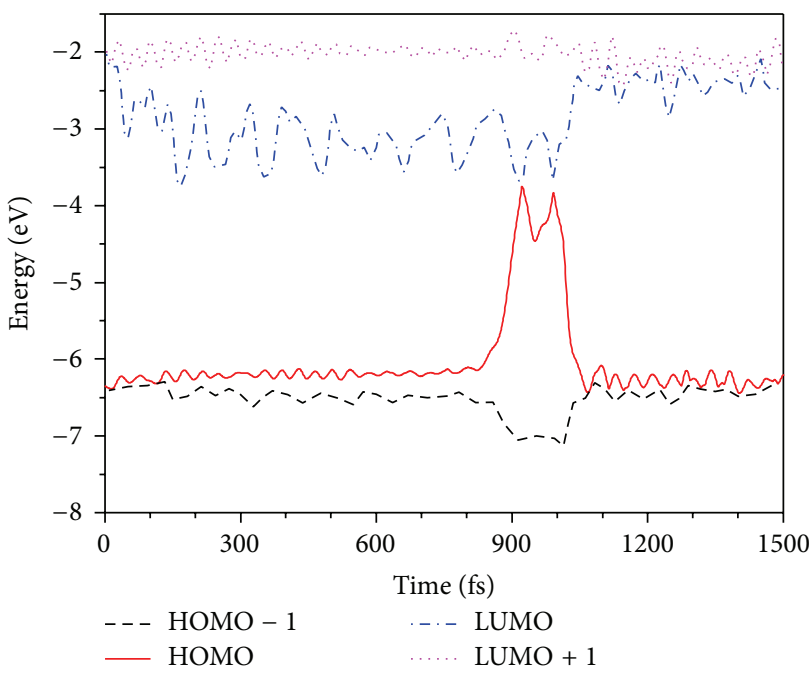

(a)

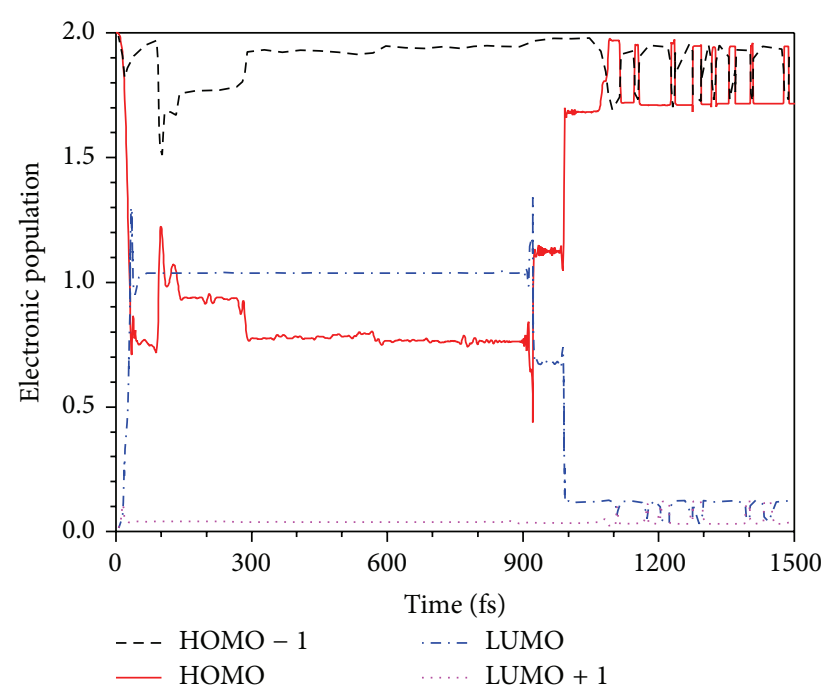

(b)

FIgure 4: (a) The variations with time, (b) the time-dependent populations of the HOMO-1, HOMO LUMO, and LUMO + 1 energies of two stacked cytosine molecules.

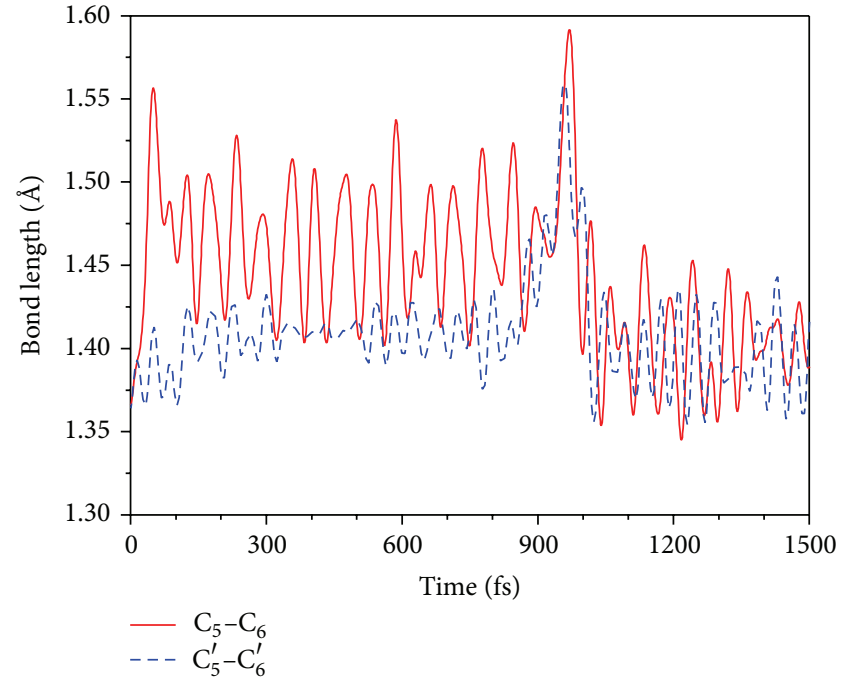

FIgURE 5: The variations with time of the lengths between the $\mathrm{C}_{5}$ and $\mathrm{C}_{6}$ atoms and the $\mathrm{C}_{5}^{\prime}$ and $\mathrm{C}_{6}^{\prime}$ atoms in two stacked cytosine molecules.

\section{Conflict of Interests}

The authors declare that there is no conflict of interests regarding the publication of this paper.

\section{Acknowledgments}

This work is supported by the National Natural Science Foundation of China (Grants no. U1330138 and no. 21203259) and the Natural Science Foundation Project of CQ CSTC (cstc2011jjA00009) and Project of the Science Technology Foundation of Chongqing Education Committee, China (nos. KJ120516 and KJ120521), and Telecommunications (no. A2012-92).

\section{References}

[1] J.-M. L. Pecourt, J. Peon, and B. Kohler, "Ultrafast internal conversion of electronically excited RNA and DNA nucleosides in water," Journal of the American Chemical Society, vol. 122, no. 38, pp. 9348-9349, 2000.

[2] N. Ismail, L. Blancafort, M. Olivucci, B. Kohler, and M. A. Robb, "Ultrafast decay of electronically excited singlet cytosine via a $\pi, \pi *$ to $\mathrm{n}_{\mathrm{O}}, \pi *$ state switch," Journal of the American Chemical Society, vol. 124, no. 24, pp. 6818-6819, 2002.

[3] M. Z. Zgierski, S. Patchkovskii, T. Fujiwara, and E. C. Lim, "The role of out-of-plane deformations in subpicosecond internal conversion of photoexcited purine bases: absence of the ultrafast decay channel in propanodeoxyguanosine," Chemical Physics Letters, vol. 440, no. 1-3, pp. 145-149, 2007.

[4] L. Serrano-Andrés, M. Merchán, and A. C. Borin, "A three-state model for the photophysics of adenine," Chemistry, vol. 12, no. 25, pp. 6559-6571, 2006.

[5] C. T. Middleton, K. de la Harpe, C. Su, Y. K. Law, C. E. CrespoHernández, and B. Kohler, "DNA excited-state dynamics: from single bases to the double helix," Annual Review of Physical Chemistry, vol. 60, pp. 217-239, 2009.

[6] Z. Pan, M. McCullagh, G. C. Schatz, and F. D. Lewis, "Conformational control of thymine photodimerization in purinecontaining trinucleotides," Journal of Physical Chemistry Letters, vol. 2, no. 12, pp. 1432-1438, 2011.

[7] M. Z. Zgierski, T. Fujiwara, and E. C. Lim, "Conical intersections and ultrafast intramolecular excited-state dynamics in nucleic acid bases and electron donor-acceptor molecules," Chemical Physics Letters, vol. 463, no. 4-6, pp. 289-299, 2008.

[8] C. E. Crespo-Hernández, B. Cohen, and B. Kohler, "Molecular spectroscopy: complexity of excited-state dynamics in DNAreply," Nature, vol. 441, no. 7094, article E8, 2006. 
[9] B. Cohen, P. M. Hare, and B. Kohler, "Ultrafast excitedstate dynamics of adenine and monomethylated adenines in solution: implications for the nonradiative decay mechanism," Journal of the American Chemical Society, vol. 125, no. 44, pp. 13594-13601, 2003.

[10] T. Takaya, C. Su, K. de la Harpe, C. E. Crespo-Hernández, and B. Kohler, "UV excitation of single DNA and RNA strands produces high yields of exciplex states between two stacked bases," Proceedings of the National Academy of Sciences of the United States of America, vol. 105, no. 30, pp. 10285-10290, 2008.

[11] C. E. Crespo-Hernández, B. Cohen, and B. Kohler, "Base stacking controls excited-state dynamics in A.T DNA," Nature, vol. 436, no. 7054, pp. 1141-1144, 2005.

[12] Y. S. Wang, O. Haze, J. P. Dinnocenzo, S. Farid, R. S. Farid, and I. R. Gould, "Bonded exciplexes. A new concept in photochemical reactions," The Journal of Organic Chemistry, vol. 72, no. 18, pp. 6970-6981, 2007.

[13] Y. Wang, O. Haze, J. P. Dinnoeenzo, S. Farid, R. S. Farid, and I. R. Gould, "Bonded exciplex formation: electronic and stereoelectronic effects," The Journal of Physical Chemistry A, vol. 112, no. 50, pp. 13088-13094, 2008.

[14] S. Yuan, W.-Y. Zhang, A.-Y. Li, Y.-M. Zhu, and Y.-S. Dou, "Dynamics simulation of a new deactivation pathway for stacked adenines," Acta Physico-Chimica Sinica, vol. 27, no. 4, pp. 825-830, 2011.

[15] Y. Dou, B. R. Torralva, and R. E. Allen, "Semiclassical electronradiation-ion dynamics (SERID) and cis-trans photoisomerization of butadiene," Journal of Modern Optics, vol. 50, no. 15-17, pp. 2615-2643, 2003.

[16] Y. Dou, B. R. Torralva, and R. E. Allen, "Interplay of electronic and nuclear degrees of freedom in a femtosecond-scale photochemical reaction," Chemical Physics Letters, vol. 392, no. 4-6, pp. 352-358, 2004.

[17] T. B. Boykin, R. Chris Bowen, and G. Klimeck, "Electromagnetic coupling and gauge invariance in the empirical tightbinding method," Physical Review B, vol. 63, no. 24, Article ID 245314, 2001.

[18] D. Porezag, T. Frauenheim, T. Köhler, G. Seifert, and R. Kaschner, "Construction of tight-binding-like potentials on the basis of density-functional theory: application to carbon," Physical Review B, vol. 51, no. 19, pp. 12947-12957, 1995.

[19] M. Elstner, D. Porezag, G. Jungnickel et al., "Self-consistentcharge density-functional tight-binding method for simulations of complex materials properties," Physical Review B, vol. 58, no. 11, pp. 7260-7268, 1998.

[20] Y. B. Lei, S. Yuan, Y. S. Dou, Y. B. Wang, and Z. Y. Wen, "Detailed dynamics of the nonradiative deactivation of adenine: a semiclassical dynamics study," Journal of Physical Chemistry A, vol. 112, no. 37, pp. 8497-8504, 2008.

[21] Y. S. Dou, S. S. Xiong, W. F. Wu, S. Yuan, and H. Tang, "Photoinduced dissociation of cyclobutane thymine dimer studied by semiclassical dynamics simulation," Journal of Photochemistry and Photobiology B: Biology, vol. 101, no. 1, pp. 31-36, 2010.

[22] W. Y. Zhang, S. Yuan, Z. J. Wang et al., "A semiclassical dynamics simulation for a long-lived excimer state of $\pi$-stacked adenines," Chemical Physics Letters, vol. 506, no. 4-6, pp. 303-308, 2011.

[23] S. Yuan, Z. Shen, W. Y. Zhang, Y. S. Dou, and G. Y. Lo, “Thymine dimer splitting in the $\mathrm{T}<>\mathrm{T}-\mathrm{G}$ trinucleotide model system: a semiclassical dynamics and TD-DFT study," International Journal of Biological Macromolecules, vol. 66, pp. 267-272, 2014.

[24] W. Zhang, S. Yuan, A. Li, Y. Dou, J. Zhao, and W. Fang, "Photoinduced thymine dimerization studied by semiclassical dynamics simulation," The Journal of Physical Chemistry C, vol. 114, no. 12, pp. 5594-5601, 2010.

[25] S. Yuan, W. Zhang, L. Liu, Y. Dou, W. Fang, and G. V. Lo, "Detailed mechanism for photoinduced cytosine dimerization: a semiclassical dynamics simulation," Journal of Physical Chemistry A, vol. 115, no. 46, pp. 13291-13297, 2011.

[26] R. E. Allen, T. Dumitrica, and B. Torralva, "Electronic and structural response of materials to fast, intence laser pluse," in Ultrafast Physical Processes in Semiconductors, K. T. Tsen, Ed., pp. 315-384, Academic Press, New York, NY, USA, 2001.

[27] W. Domcke, D. R. Yarkony, and H. Köppel, Conical Intersections: Electronic Structure, Dynamics \& Spectroscopy, World Scientific Publishing, Singapore, 2004.

[28] M. Baer, Beyond Born-Oppenheimer: Electronic Nonadiabatic Coupling Terms and Conical Intersections, Wiley-Interscience, Hoboken, NJ, USA, 2006.

[29] M. J. Bearpark, F. Bernardi, S. Clifford, M. Olivucci, M. A. Robb, and T. Vreven, "Cooperating rings in cis-stilbene lead to an S0/S1 conical intersection," The Journal of Physical Chemistry A, vol. 101, no. 21, pp. 3841-3847, 1997.

[30] B. G. Levine and T. J. Martínez, "Isomerization through conical intersections," Annual Review of Physical Chemistry, vol. 58, pp. 613-634, 2007. 

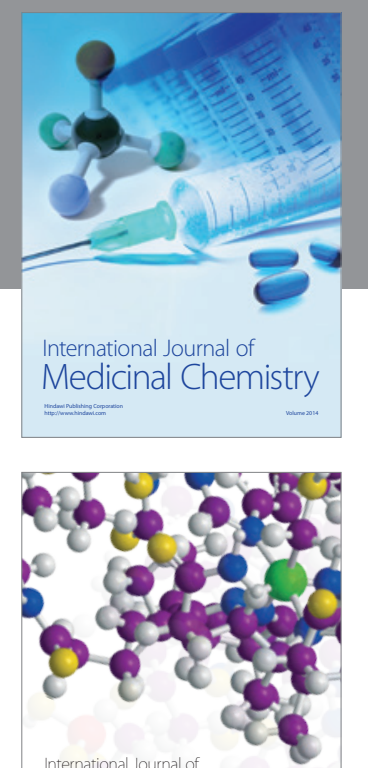

\section{Carbohydrate} Chemistry

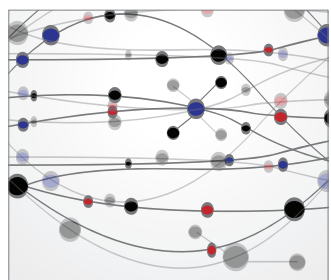

The Scientific World Journal
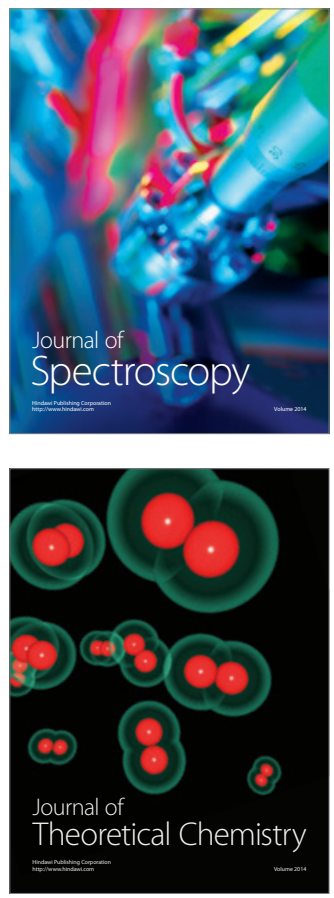
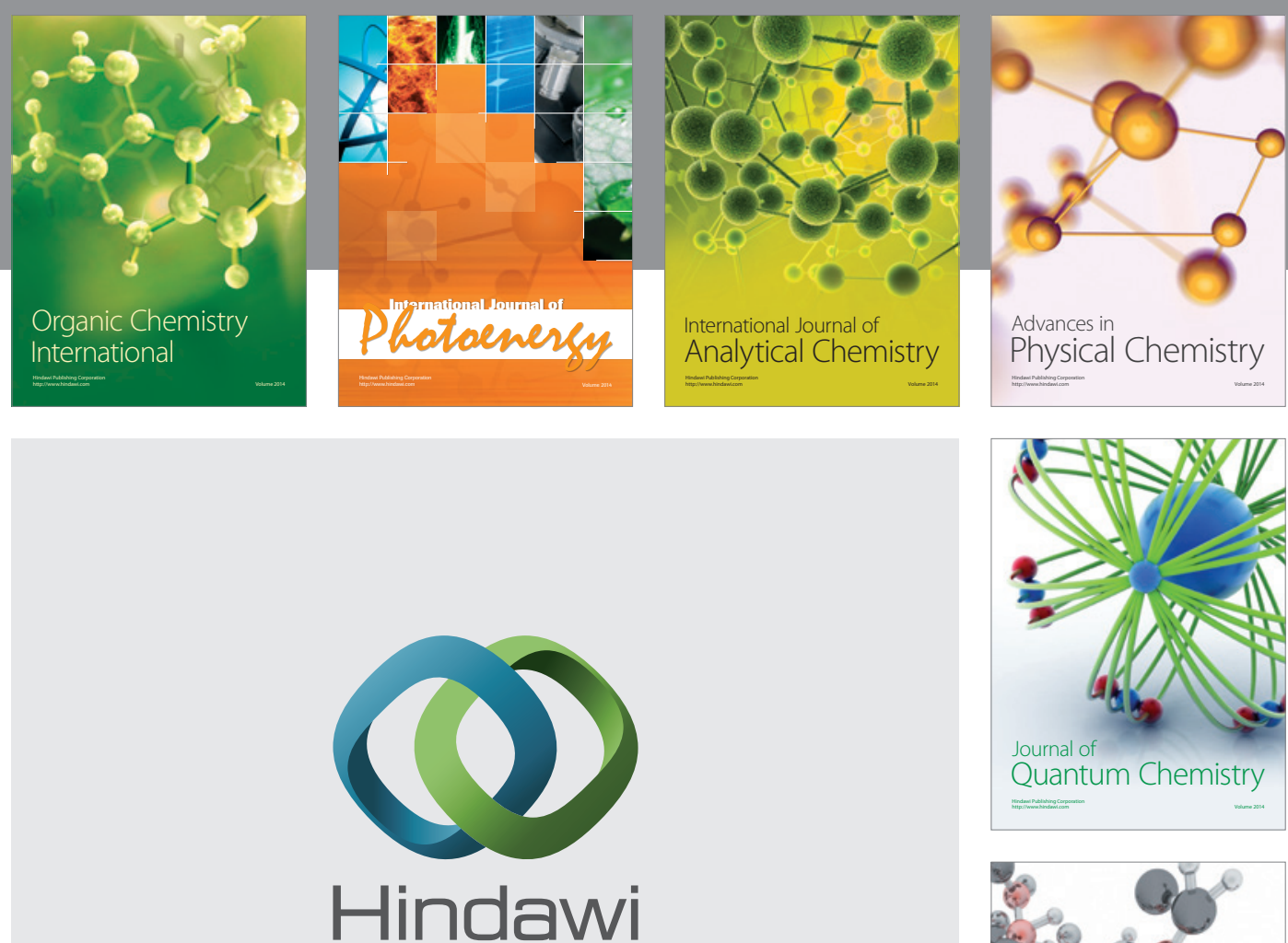

Submit your manuscripts at

http://www.hindawi.com

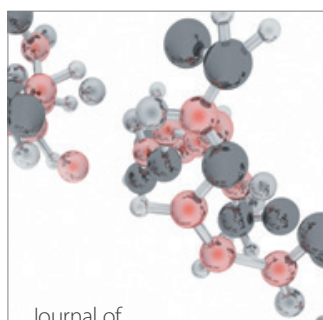

Analytical Methods

in Chemistry

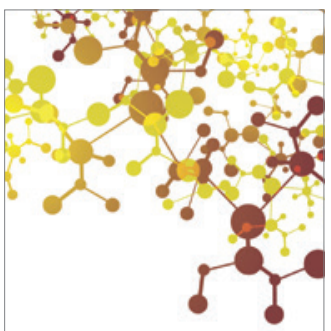

Journal of

Applied Chemistry

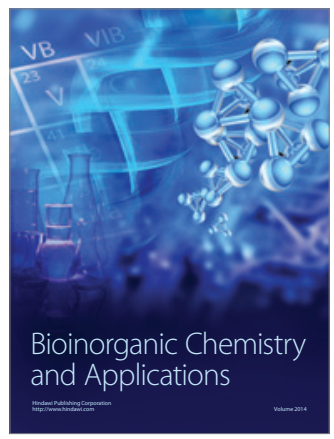

Inorganic Chemistry
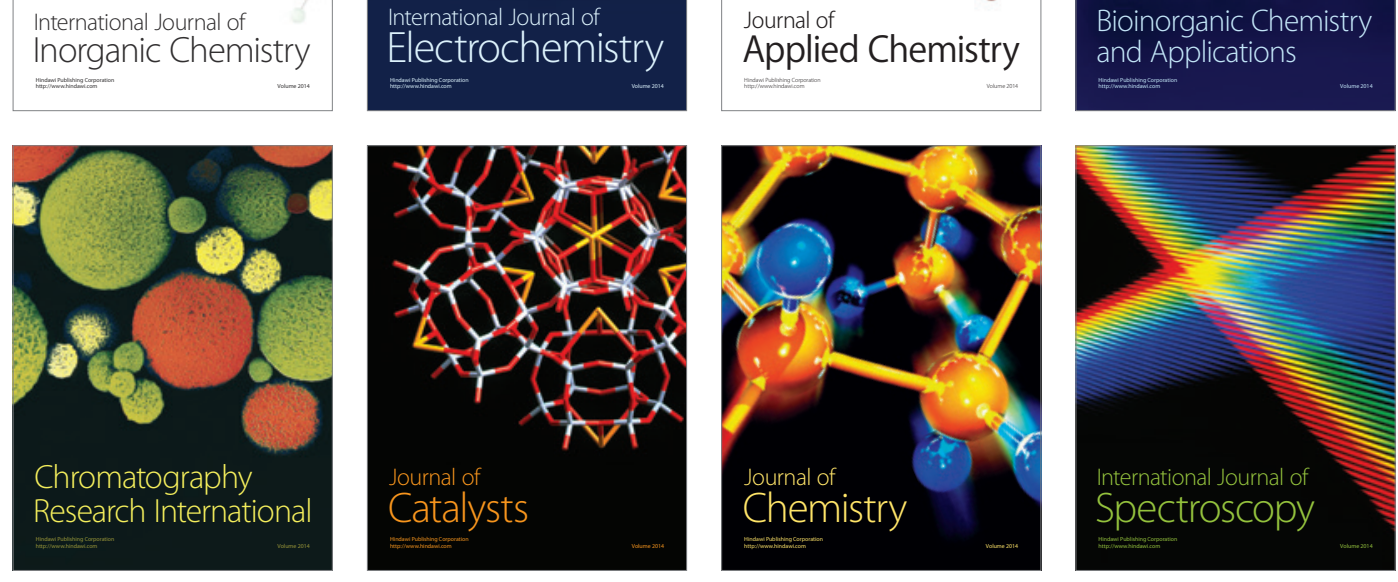